\title{
Introduction to the RE'14 special issue
}

\author{
Robyn R. Lutz ${ }^{1}$
}

Received: 12 April 2015/Accepted: 13 April 2015/Published online: 23 April 2015

(C) Springer-Verlag London 2015

This special issue of the Requirements Engineering Journal (REJ) contains extended versions of selected papers from the 22nd IEEE International Requirements Engineering Conference (RE'14). This continues a long-standing collaboration between RE and REJ to invite the authors of the best papers presented at the conference to submit extended papers to reach the wider journal readership. As program chair of RE' 14 , it is my pleasure to introduce these papers to you.

The conference was held at the Blekinge Institute of Technology in Karlskrona, Sweden, August 25-29, 2014, with Tony Gorschek as general chair, Sarah Gregory and Marjo Kauppinen as industry co-chairs, and Jüergen Börstler as organizing chair. Three hundred people registered for the main conference, and over 420 registered in total for the conference and its associated tutorials, doctoral symposium, and workshops. The conference featured stirring keynotes by Anthony Wasserman, Annie Antón, and Anthony Finkelstein. RE'14 also offered a variety of panels, mini-tutorials, lightning-round poster and tool demonstrations, an Industry Lab, and multiple social activities to foster new ideas, uses, and collaborations in requirements engineering.

The theme of RE'14 was innovation. Innovation in requirements engineering explores new ideas and breakthroughs in the area. Innovation through requirements engineering describes how its practices, processes, models, methods, and frameworks enable and support innovation in product development.

Robyn R. Lutz

rlutz@iastate.edu

1 Department of Computer Science, 226 Atanasoff Hall, Iowa State University, Ames, IA 50011, USA
RE'14 received 115 submissions to its research track by authors from 30 countries. Following review by three members of the program committee and an online discussion among the reviewers of each paper, the program board met and used the reviews and discussion to select 31 research papers for presentation at the conference, an acceptance rate of $27 \%$. I extend my thanks to the program committee and the program board for their service, and to the authors for their submissions. The high quality of the authors' papers provided the strong technical program for RE'14, and that is reflected in the papers offered here.

The three papers published in this issue are extended versions of the work invited from the best of those RE'14 research-track papers. Each of these extended papers underwent rigorous, additional review by three reviewers in accordance with the REJ review process before acceptance to the special issue.

The first paper is "TiQi: Answering Unstructured Natural Language Trace Queries" by Piotr Pruski, Sugandha Lohar, William Goss, Alexander Rasin, and Jane ClelandHuang. Developers often find it hard to specify complex trace queries, which limits their access to the traceability information they need. In response to this problem, the authors have developed TiQi, a process to automatically translate unstructured spoken or written natural language queries into executable SQL (structured query language) statements. TiQi is supported by a database query mechanism as well as by domain- and project-specific elements. Experimental results show that users prefer written natural language queries over spoken queries. In experiments on two datasets, TiQi's accuracy rate in translating queries is shown to vary from 47 up to $93 \%$. Two additional experiments evaluated the performance of TiQi's ambiguity resolution and what the sample size of the trace query collection needs to be to construct a 
relatively complete traceability information model. These results significantly advance the auto-generation of SQL trace queries from written natural language queries in accordance with user preferences.

The second paper is "Rationalism with a Dose of Empiricism: Combining Goal Reasoning and Case-Based Reasoning for Self-Adaptive Software Systems" by Wenyi Qian, Xin Peng, Bihuan Chen, John Mylopoulos, Huanhuan Wang, and Wenyun Zhao. A challenge to self-adaptive systems that use a runtime requirements model to make decisions is that, in complex systems, relationships between system configurations, environment, and requirements may not be precisely enough specified to make the right decision. In this paper, the authors report development of an adaptive approach that combines case-based reasoning and goal reasoning to address this problem. Previous successful adaptations, including information about the system configuration at the time, are kept and reused when possible. When no similar previous case exists, then goal reasoning is deployed. To avoid being trapped in a suboptimal adaptation, the authors introduce a case mutation mechanism. In a study on an online shopping system, their approach outperforms either the case-based or goal reasoning approach alone. More generally, the authors identify trends in how solutions for an adaptation problem evolve. Progress in the quality of decisions made by selfadaptive systems, such as the approach presented here, will be essential to the success of requirements-driven runtime adaptations.

The third paper is "Leveraging Topic Modeling and Part-of-Speech Tagging to Support Combinational
Creativity in Requirements Engineering" by Tanmay Bhowmik, Nan Niu, Juha Savolainen, and Anas Mahmoud. The authors present a framework for promoting creativity in requirements generation. To achieve this, they have developed an end-to-end automated process for extracting familiar ideas from the requirements and comments of stakeholder groups of an existing software system, and then connecting these ideas in unfamiliar combinations to yield new candidate requirements. The paper describes how new combinations of verbs and nouns, where each word in the pair is associated with the dominant topic of a different stakeholders' group, successfully yield unfamiliar combinations of familiar topics for elaboration into innovative requirements. The authors report promising results from application to two large, open-source software systems and in controlled studies of the feasibility of their automated, combinational creativity framework. The paper highlights the crucial role that creativity can play in identifying requirements that better meet users' needs, and it offers a framework combining automation and interaction in support of that vision.

I thank both the authors of the invited papers and the reviewers. I also thank Pericles Loucopoulos, the REJ Editor-in-Chief, and the REJ editorial team for their assistance in making this special issue possible and for continuing the collaboration between RE and REJ.

Looking ahead, RE'15 takes place in Ottawa, Canada, and RE' 16 in Beijing, China. I hope that you can join us for those conferences. 